OPEN ACCESS

Edited by:

Qinghua He,

Southwest University, China

Reviewed by:

Valentina Cazzato,

Liverpool John Moores University,

United Kingdom

Ofir Turel,

California State University, Fullerton, United States

*Correspondence:

Carmelo M. Vicario

carmelo.vicario@utas.edu.au

Specialty section:

This article was submitted to

Psychopathology,

a section of the journal

Frontiers in Psychiatry

Received: 23 September 2017

Accepted: 22 March 2018

Published: 09 April 2018

Citation:

Vicario CM and Felmingham K (2018)

The Perception of Time Is

Underestimated in Adolescents With

Anorexia Nervosa.

Front. Psychiatry 9:121.

doi: 10.3389/fpsyt.2018.00121

\section{The Perception of Time Is} Underestimated in Adolescents With Anorexia Nervosa

\author{
Carmelo M. Vicario ${ }^{1,2,3 *}$ and Kim Felmingham ${ }^{4}$ \\ 'School of Psychology, University of Tasmania, Hobart, TAS, Australia, ${ }^{2}$ Dipartimento di Scienze Cognitive, Psicologiche, \\ Pedagogiche e degli Studi Culturali, Messina, Italy, ${ }^{3}$ Department of Psychology and Neurosciences Leibniz Research Center \\ for Working Environment and Human Factors, Dortmund, Germany, ${ }^{4}$ School of Psychological Sciences, University of \\ Melbourne, Parkville, VIC, Australia
}

Research has revealed reduced temporal discounting (i.e., increased capacity to delay reward) and altered interoceptive awareness in anorexia nervosa (AN). In line with the research linking temporal underestimation with a reduced tendency to devalue a reward and reduced interoceptive awareness, we tested the hypothesis that time duration might be underestimated in AN. Our findings revealed that patients with AN displayed lower timing accuracy in the form of timing underestimation compared with controls. These results were not predicted by clinical, demographic factors, attention, and working memory performance of the participants. The evidence of a temporal underestimation bias in AN might be clinically relevant to explain their abnormal motivation in pursuing a long-term restrictive diet, in line with the evidence that increasing the subjective temporal proximity of remote future goals can boost motivation and the actual behavior to reach them.

Keywords: anorexia nervosa, time processing, time underestimation, long-term restrictive diet, symptomatic trait

\section{INTRODUCTION}

Anorexia nervosa (AN) is a disorder of unknown etiology, characterized by severe eating restriction and distorted body image (1), which mainly affects young women (2). The estimated incidence of this disorder in the population is around 8 per 100,000 persons per year (3), while the mortality rate is the highest of any psychiatric disorder (4).

An excessive self-control linked to reward processing is considered one hallmark symptom in AN [e.g., Ref. (5-7)]. This is also suggested by recent investigations (8-10) using temporal discounting paradigms referring to a monetary reward (i.e., participants were asked to choose between smallersooner and larger-later monetary rewards) that examine the level to which a reward is devalued (discounted) over time (11). In particular, this research has revealed a lower devaluing associated with delayed reward in AN compared with healthy controls AN had significantly lower discount rates (i.e., less steep discounting) in the intertemporal choice task (10). In other words, as explained by Steinglass et al. (8), one dollar in 3 months was worth more for the AN group than it was for the healthy controls group.

Such reduced temporal discounting in AN has been interpreted as proof of enhanced ability (thus higher self-control) to delay a reward, which might help to explain their capacity to maintain a food restriction for a long time, possibly in favor of a future, more attractive reward-i.e., a further weight loss (8). However, the literature is not consistent as no temporal discounting difference between AN and controls has been documented by others [i.e., Ref. (11-13)]. These contrasting results have been explained by methodological discrepancies in task design and/or age of participants $(12,13)$. 
In research on temporal discounting in healthy populations, several scientists have suggested the importance of separating the perception of the value associated with a reward from the perception of a delay in temporal discounting [e.g., Ref. (14-17)]. In support of this suggestion, a study by Kim and Zauberman (18) has shown that the level of overall time contraction (i.e., how long or short individuals perceive time horizons to be overall) contributes to the degree of hyperbolic discounting-i.e., the tendency to choose a smaller-sooner reward over a larger-later reward. These authors found that the individual levels of hyperbolic discounting were positively correlated with the participants' time estimation length, i.e., the longer the time estimation, the higher the tendency to choose a smaller-sooner reward over a larger-later reward. This result has been confirmed in a subsequent work showing that people who overestimate the passage of time hold less value in delayed rewards (19). Moreover, a study by Suo et al. (20) found lower choice percentage for the smaller-immediate reward in participants who tended to underestimate time, compared with participants who tended to overestimate time. Taken together, the evidence of a link between temporal discounting rate and the subjective experience of time suggests a specific prediction about the perception of time in AN. In particular, one might expect a tendency of individuals with AN to underestimate the duration of temporal intervals, as an effect of a reduced temporal discounting in this clinical population $(8-10,21)$.

Dysfunction in the ability to perceive time duration in individuals with AN can also be predicted in relation to their altered interoceptive functions [e.g., Ref. (22)], in line with evidence that time perception is modulated by interoception [e.g., Ref. (23-25)]. Meissner and Wittmann (26) have found that time estimation accuracy correlates with both the slope of cardiac slowing during the perception of temporal intervals and the conscious awareness of an individual's own heart beats. Moreover, Di Lernia et al. (27) have recently found a temporal underestimation of the duration of interoceptive stimuli in relation to a diminished processing of high salience stimuli from the body. This latter work further supports the prediction of a temporal underestimation in $\mathrm{AN}$, given the evidence of altered processing of interoceptive (22) and body-related (28) information in this clinical population.

The hypothesis that AN might be associated with dysfunctional time estimation is also supported by the neuroimaging literature. Structural and functional data have shown that the frontostriatal pathway, which is known to play a key role in the experience of time [e.g., Ref. (29-33)], is the most susceptible neural structure to cognitively maintained restraint of appetite in AN [Ref. (34), see also Ref. (35), for a review]. The insula is another region considered putatively important for explaining the pathophysiology of individuals with AN [Ref. (32, 33, 35-38) for a review], and the research has demonstrated its direct involvement in time keeping functions [e.g., Ref. (39-41); see Ref. (42) for a review]. The neural factors predictive of potential time keeping alterations in $\mathrm{AN}$ include dysfunction in the dopaminergic system, which is also directly implied in the regulation of the mental clock's beats [e.g., Ref. $(30,43-46)]$. Evidence of dysregulation in dopaminergic processes in AN is provided by a PET study in subjects who recovered from AN (47). These authors found increased dopamine (i.e., D2/D3) receptor (DRD3) binding in the ventral striatum, a region that modulates responses to reward stimuli (48) and the subjective experience of time $(49,50)$. Moreover, research suggests a greater dopamine model reward-learning signal in the anteroventral striatum, insula, and in the prefrontal cortex of AN patients (51).

From a clinical point of view, the evidence of a timing alteration in individuals with AN might be helpful in explaining the dietary restriction associated with AN. A time underestimation bias might contribute to the abnormal motivation of AN in delaying or skipping the consumption of a meal (a primary reward) for a longer-term more desirable reward (i.e., the weight loss). In presence of such a bias, individuals with AN might mentally represent their main goal (i.e., losing more weight) as more temporally proximal than how it is in reality. This could explain their strong motivation in keeping a long-term restrictive diet. An alternative, not mutually exclusive, suggestion is that a temporal underestimation might cause a misperception of meal duration and between-meal epochs. In this regard, one might assume that if between-meal intervals seem shorter than they actually are, then the motivation for the next meal is reduced. Evidence in support for such arguments exists in research on obesity (e.g., people eat more often when the interval between meals seem longer), smoking, and technology use (52-55). Interestingly, the use of a temporal discounting paradigm in previous studies in AN did not allow clarifying whether the reduced discounting response selectively reflect the value of the delayed reward, or if it might also be linked to the encoding of temporal information emerging from the execution of such a paradigm. Therefore, testing AN with an explicit timing task might also address this timely question.

Based on this literature, in this study, we tested if there was a time processing deficit in AN by using a supra-second time estimation task of visual stimuli. We also investigated any role played by attention and working memory (WM) skills, which are known to be predictive of temporal performance in healthy humans and clinical populations $(30,56-62)$. Moreover, we explored the contribution of depression, stress, and anxiety symptoms, which might influence time keeping skills [e.g., Ref. (63)].

\section{PARTICIPANTS}

Data from individuals with $\mathrm{AN}$ and healthy controls were extracted from the Brain Resource International Database $\left(B_{R} D^{1}\right)$. This database contains data from multiple laboratories (New York, Rhode Island, Nijmegen, London, Adelaide, and Sydney) that have been acquired using standardized data acquisition techniques for cognitive tasks (IntegNeuro) including the time estimation task. Inter-lab reliability and test-retest reliability measures are high as documented in previous works [e.g., Ref. (64-66)]. The request of data is only granted to scientists who are formally registered to BRID. Access to the database was approved after the evaluation of our research proposal on time perception in AN. The review of our proposal was executed by other colleagues formally registered to BRID. After formal approval, the

${ }^{1}$ http://www.brainnet.net/about/governance-and-management/. 
manager of the database provided an excel copy of all the available data on $\mathrm{AN}$ and control participants.

The AN participants were recruited from two adolescent inpatient eating disorder programs at associated teaching hospitals of the University of Sydney, Australia (The Children's Hospital at Westmead and Westmead Hospital). Specific inclusion criteria for AN participants included DSM-IV diagnosis of AN, female, aged between 12.5 and 17.5 years and in their first hospital admission at the time of recruitment. The exclusion criteria of this database included a personal or family history of mental illness, brain injury, neurological disorder, serious medical condition, drug/ alcohol addiction, first-degree relative with bipolar disorder, schizophrenia, or genetic disorder, no history of bulimia nervosa, or BMI above 17.5 on day of baseline assessment. We adopted the 10th percentile of the body mass index as a cutoff for underweight in the control group (67). From two initial samples of 41 patients and 41 controls, we excluded participants with less than 8 years of education, to make this variable equivalent between the two samples. Furthermore, we did not include participants with missing information with regard to BMI, and participants providing outlier performance (i.e., $\pm 3 \mathrm{SD}$ ). Therefore, the final analysis included a sample of $30 \mathrm{AN}$ patients (mean age $=15.43 \pm 1.60$ $\mathrm{SD}$ ) and 21 healthy controls (mean age $=15.32 \pm 1.81$ ). All participants gave written informed consent to participate in the study. The study was approved by the University of Tasmania, School of Psychology, Research Ethics Committee.

\section{METHOD}

\section{Psychometric Measures}

Anorexia nervosa diagnosis was made using the clinicianadministered DSM-IV criteria (2). To estimate disorder severity and to assess for eating disorder-related psychopathology, individuals completed the Eating Disorders Inventory-3 (68). WM and attention performance were examined by using, respectively, the Digit Span (69) and the Switching of Attention (70) tasks. To assess for depressed, anxious, and stressed mood, the depression anxiety and stress scale (DASS) was administered (71). A detailed description of the psychometric measures is provided in the following paragraphs.

\section{TASKS AND PROCEDURE}

Participants were seated in a sound attenuated room in front of a touchscreen computer (NEC MultiSync LCD 1530V). All participants completed the cognitive tests as part of a reliable and valid computerized test battery $(64,66)$. Tests were administered using prerecorded task instructions (via headphones) and computerized and voice recording was used for answers. All participants performed a practice trial before the formal completion of the proposed tasks. Responses were provided by using the touchscreen.

\section{Digit Span Task}

Participants were presented with a series of digits on the touchscreen, separated by a $1-s$ interval. The subjects were then immediately asked to enter the digits on a numeric keypad on the touchscreen. In the first part of the test, subjects were required to recall the digits in forward order and reverse order in the second. In each part, the number of digits in each sequence was gradually increased from 3 to 9, with two sequences at each level. The dependent measure was the total number of correct trials forward and backward.

\section{Switching of Attention Task}

This modified version of the Trail Making Test consisted of two parts. The first, a measure of psychomotor speed, required the connecting of numbers in ascending sequence (i.e., 1-2-3-, etc.) (Switching of Attention - Number). The second, requiring speeded cognitive flexibility, asked participants to connect numbers and letters in an ascending but alternating sequence (i.e., 1-A-2-B, etc.) (Switching of Attention - Number/Letter). Time for completion for each part served as dependent variables.

\section{The Time Estimation Task}

A black circle appeared on the screen, turning green for times varying between 1 and $12 \mathrm{~s}$, in steps of $1 \mathrm{~s}$, in pseudo-random order and for a total of 12 intervals. Therefore, the number of trials was 12. Each participant was required to attend to the screen and estimate the duration of the target trace on the screen, using keys on a fixed display touchpad at the bottom of the screen with the duration range between 1 and $12 \mathrm{~s}$. Each temporal switch was presented once. Task duration was approximately $3 \mathrm{~min}$ (see Figure 1 for a schematic representation of the task execution). The task assesses the ability to estimate time intervals without a clock and relates to the ability to pre-plan actions, decide temporal onset, monitor the time course of initiation, and anticipate

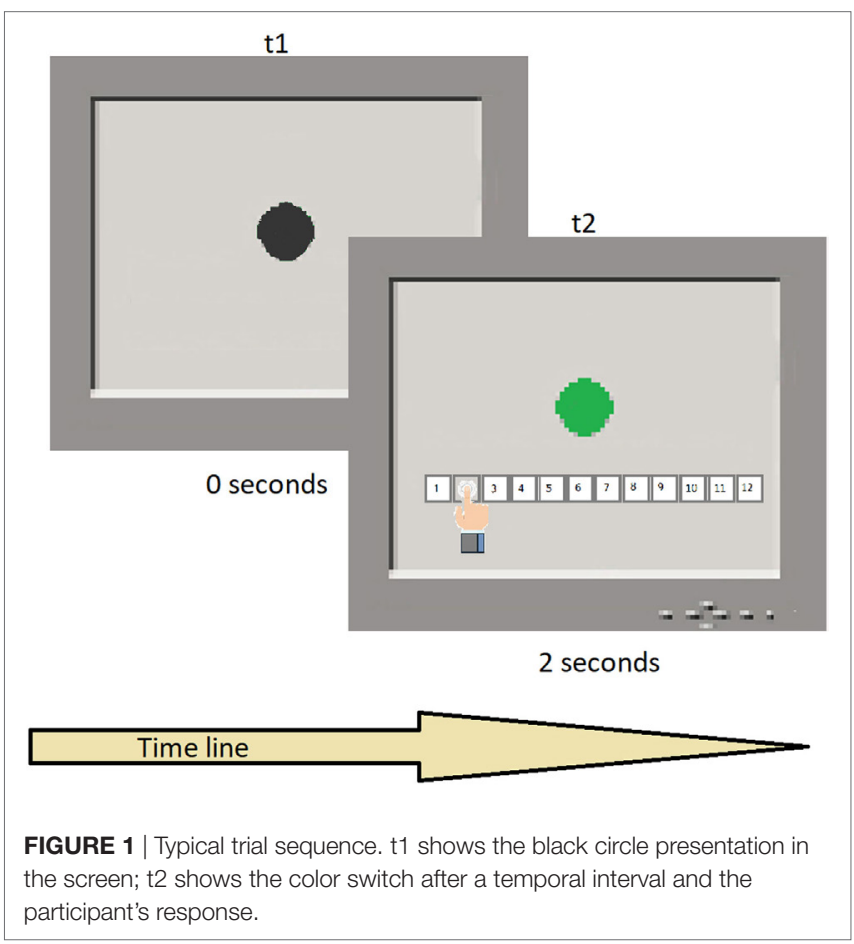


outcomes. For more information about the task, refer to Block et al. (72) and Gunstad et al. (73).

\section{DATA ANALYSIS}

Participants' task performance was evaluated by considering the proportional bias $(\mathrm{PB})$, which provides a measure of the estimation accuracy calculated from the 12 temporal intervals, where the bias for each trial is calculated as a positive or negative percentage of the actual presented interval. PB is estimated from the absolute value of the average difference between the actual duration of the stimulus and the user-estimated duration, weighted by the length of the stimulus. Thus, an overall positive score (i.e., $>0$ ) indicates a temporal overestimation; while an overall negative score (i.e., <0) indicates a temporal underestimation. As post hoc analysis, we also calculated the estimation bias variability (EBV), which represents the SD average of the PB. Timing variability measure reflects how repeated responses are scattered from their target within a particular experimental condition. We have calculated this parameter as a further exploratory measure, with the purpose to explore participants' variability in detecting the duration of temporal intervals.

Proportional bias and EBV scores of our clinical and control samples were compared by using pairwise $t$-test comparisons. Although we were interested in exploring any role of other variables on time keeping skills, we choose the $t$-test comparison, instead of the analysis of covariance (ANCOVA), in keeping with the suggestion of a recent work (74) that discourages the use of ANCOVA in the case of classification designs, that is designs that indicate a comparison of different populations such as in our case. The participants $\mathrm{PB}$ scores were also compared against the 0 score via one sample $t$-test, to investigate if the timing performance of our two samples significantly deviated from the 0 score, which reflects the timing performance in absence of biases. Finally, Pearson correlation analyses were implemented to measure any relationship between the time estimation performance and the cognitive/affective measures collected for our participants. We run separate analyses for $\mathrm{AN}$ and controls as the intent of our research was exploring any between groups difference with regard to the several variables included in our research.

The $p$ value was adjusted with Bonferroni correction, as PB and EBV scores were compared with 14 variables (75). Therefore, the corresponding level of significance for the correlation outputs is $<0.003$ (i.e., $0.05 / 14$, see Table 2 for details). Data analysis was performed using Statistica software, version 8.0, Stat Soft, Inc., Tulsa, OK, USA.

\section{RESULTS}

The sample size was sufficiently large (i.e., $\geq 46$ ) for the related effect size of Cohen's $d=0.75$, a statistical power of 0.8 and a probability level of 0.05 . The observed post hoc statistical power was 0.833 . All these analyses were implemented via Free Statistics Calculator. ${ }^{2}$ Table 1 presents a summary of means and SDs on demographic, cognitive, and clinical variables for the groups and test statistics for between group comparisons (i.e., pairwise $t$-test). A significant between groups difference was reported for all clinical measures and the BMI score, while no difference was reported for cognitive and demographic variables (Table 1 for details).

The $t$-test on timing performance documented a significant between group difference for the $\mathrm{PB}$ score, which was lower for the AN sample $(M=-0.072, S D=0.102)$, compared with controls $(\mathrm{M}=0.027, \mathrm{SD}=0.152)(t=-2.80, p=0.007$, Cohen's $d=0.75)$, see Figure 2 for details. The one sample $t$-test relative to 0 revealed a significant difference for the AN sample $(t=-3.890$, $p<0.001$, Cohen's $d=0.69$ ). By contrast, no significant difference from the 0 score was reported comparing the performance of the control group $(t=0.817, p=0.423$, Cohen's $d=0.20)$. Finally, we did not find a significant difference for the EBV score $(p=0.121)$.

The correlation analyses of AN and control participants did not reveal significant results according the adjusted $p$-level (see Table 2 for details).

${ }^{2}$ https://www.danielsoper.com/statcalc/calculator.aspx?id=49.

TABLE 1 | The table reports the means and the $t$-test scores for the examined clinical and cognitive affective performance associated to AN and control participants.

\begin{tabular}{|c|c|c|c|c|}
\hline & Mean-AN & Mean-controls & $t$-Test & $p$-Level \\
\hline Age & $M=15.43, S D=1.66$ & $M=15.33, S D=1.81$ & 0.217 & 0.828 \\
\hline Education & $M=10.63, S D=1.62$ & $M=10, S D=1.70$ & 1.341 & 0.186 \\
\hline Depress & $M=9.72, S D=5.90$ & $M=2.47, S D=2.85$ & $4.904^{\star}$ & $<0.001$ \\
\hline Anxiety & $M=5.88, S D=4.12$ & $M=1.23, S D=1.17$ & $4.871^{\star}$ & $<0.001$ \\
\hline Stress & $M=8.52, S D=4.98$ & $M=3.42, S D=3.12$ & $3.810^{\star}$ & $<0.001$ \\
\hline Digitot & $M=6.25, S D=2.90$ & $M=6.70, S D=2.16$ & 0.228 & 0.820 \\
\hline Digitsp & $M=5.74, S D=1.60$ & $M=5.92, S D=1.32$ & 0.261 & 0.794 \\
\hline Rdigitot & $M=4.51, S D=2.37$ & $M=3.88, S D=2.29$ & 1.638 & 0.107 \\
\hline Rdigitsp & $M=4.71, S D=1.91$ & $M=4.40, S D=1.52$ & 1.505 & 0.138 \\
\hline Swoadur1 & $M=18,656, S D=4,260$ & $M=19,652, S D=4,155$ & -1.799 & 0.081 \\
\hline Swoaerr1 & $\mathrm{M}=0.80, \mathrm{SD}=1.28$ & $M=0.66, S D=0.960$ & 0.240 & 0.811 \\
\hline Esoadur2 & $M=40,276 \cdot 1, S D=13,273 \cdot 2$ & $M=41,076.1, S D=13,057$ & -1.070 & 0.289 \\
\hline Esoaerr2 & $M=1.02, S D=2.00$ & $M=1.29, S D=1.56$ & -0.872 & 0.387 \\
\hline $\mathrm{BMl}$ & $M=16.10, S D=1.07$ & $M=21.11, S D=4.01$ & $-6.485^{\star}$ & $<0.001$ \\
\hline
\end{tabular}

${ }^{*}$ A significant result.

Digitot (digit span forward, correct trials); Digitsp (digit span forward, recall span); Rdigitot (digit span reverse, correct trials); Rdigitsp (digit span reverse, recall span); Swoadur1 (switching of attention, completion time-digits); Swoaerr1 (switching of attention, errors-digits); Esoadur2 (switching of attention, completion time-digits + letters); Esoaerr2 (switching of attention, errors - digits + letters); BMI (body mass index). 
TABLE 2 | The table provides details about the correlation results between the PB and estimation bias variability (EBV) scores of the AN and the control groups and their performance with regard to clinical and cognitive measures.

\begin{tabular}{|c|c|c|c|c|c|c|c|c|}
\hline & \multicolumn{2}{|c|}{ PB AN } & \multicolumn{2}{|c|}{ EBV $A N$} & \multicolumn{2}{|c|}{ PB controls } & \multicolumn{2}{|c|}{ EBV controls } \\
\hline & $\boldsymbol{R}$ & $p$-Level & $\boldsymbol{R}$ & $p$-Level & $\boldsymbol{R}$ & $p$-Level & $\boldsymbol{R}$ & $p$-Leve \\
\hline Age & -0.289 & 0.120 & -0.114 & 0.546 & -0.202 & 0.378 & -0.0381 & 0.869 \\
\hline Education & -0.306 & 0.100 & -0.063 & 0.738 & -0.270 & 0.236 & -0.117 & 0.611 \\
\hline Depress & -0.135 & 0.476 & -0.052 & 0.782 & -0.273 & 0.230 & -0.234 & 0.307 \\
\hline Anxiety & -0.102 & 0.588 & 0.054 & 0.775 & -0.035 & 0.880 & -0.023 & 0.921 \\
\hline Stress & -0.059 & 0.754 & -0.011 & 0.952 & -0.120 & 0.601 & -0.204 & 0.373 \\
\hline Digitot & 0.397 & 0.032 & -0.460 & 0.012 & -0.181 & 0.430 & 0.292 & 0.198 \\
\hline Digitsp & 0.427 & 0.020 & -0.495 & 0.006 & -0.361 & 0.107 & 0.142 & 0.537 \\
\hline Rdigitot & 0.167 & 0.385 & -0.290 & 0.126 & -0.298 & 0.188 & 0.357 & 0.111 \\
\hline Rdigitsp & 0.157 & 0.413 & -0.292 & 0.123 & -0.293 & 0.197 & 0.257 & 0.259 \\
\hline Swoadur1 & 0.171 & 0.365 & 0.001 & 0.999 & 0.018 & 0.9350 & -0.072 & 0.754 \\
\hline Swoaerr1 & 0.099 & 0.601 & 0.271 & 0.146 & 0.158 & 0.493 & 0.314 & 0.165 \\
\hline Esoadur2 & -0.024 & 0.898 & 0.472 & 0.008 & 0.217 & 0.343 & -0.023 & 0.919 \\
\hline Esoaerr2 & 0.312 & 0.092 & 0.427 & 0.018 & 0.3100 & 0.170 & 0.191 & 0.406 \\
\hline $\mathrm{BMI}$ & 0.053 & 0.779 & 0.019 & 0.919 & -0.304 & 0.178 & 0.075 & 0.746 \\
\hline
\end{tabular}

Digitot (digit span forward, correct trials); Digitsp (digit span forward, recall span); Rdigitot (digit span reverse, correct trials); Rdigitsp (digit span reverse, recall span); Swoadur1 (switching of attention, completion time-digits); Swoaerr1 (switching of attention, errors-digits); Esoadur2 (switching of attention, completion time-digits + letters); Esoaerr2 (switching of attention, errors - digits + letters); BMI (body mass index).

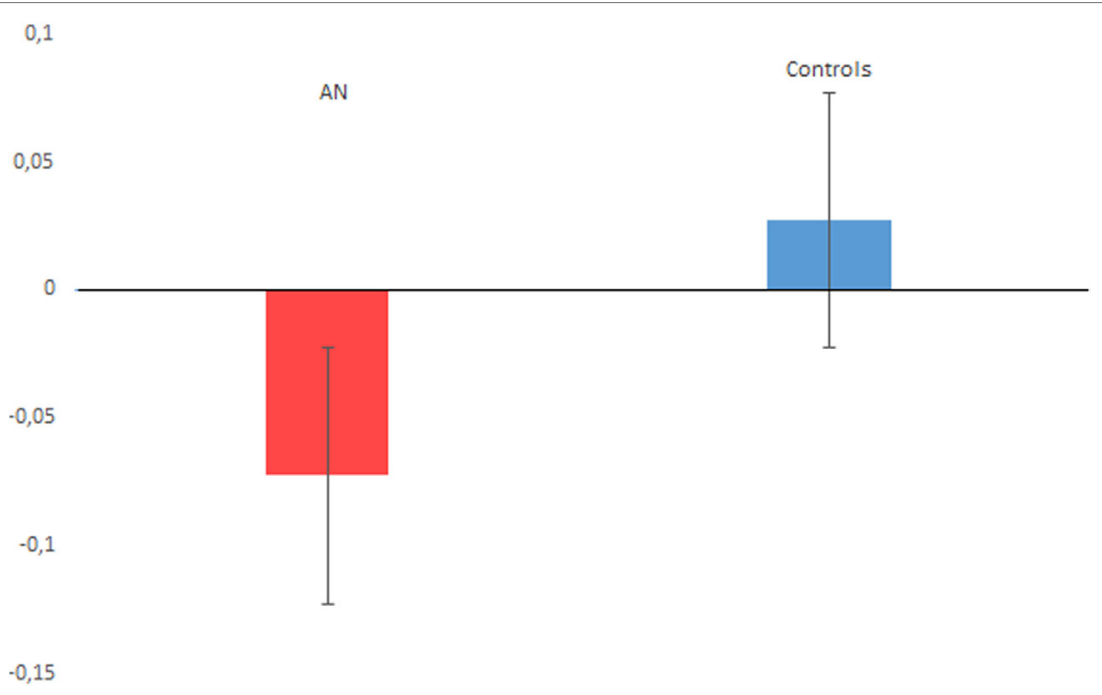

FIGURE 2 | The figure plots the proportional bias performance associated with anorexia nervosa (AN) and control participants. A negative value indicates time underestimation, while a positive value indicates time overestimation. Vertical bars denote \pm SEs.

\section{DISCUSSION}

In this study, we provided evidence that time perception is underestimated in AN. This result is in agreement with our initial hypothesis based on the research documenting temporal underestimation in the presence of reduced temporal discounting (20) and altered processing of interoceptive/body information (27), which have been documented in AN [e.g., Ref. $(8,10,22,28)]$. Moreover, the timing performance of the AN group significantly deviated from the 0 score of the $\mathrm{PB}$ measure, where the 0 score represents the timing performance in absence of any bias. Therefore, the AN timing performance can also be described as being less accurate than that of the control sample. This result can be explained in relation to the reduced cardiac awareness in $\mathrm{AN}(76,77)$. In fact, the lower the awareness of individual heart beats, the lower the time estimation accuracy (26). However, we did not measure cardiac awareness in our AN participants, and therefore this suggestion remains to be directly tested in future works. As outlined in the Section "Introduction," a possible implication of a temporal underestimation bias in $\mathrm{AN}$ is that these patients might perceive their forthcoming goals-such as losing more weight-as more temporally proximal than how they are in the reality. This might contribute to explaining their heightened motivation in keeping a long-term restrictive diet. Such an interpretation is in line with the evidence (78) that increasing the subjective temporal proximity of remote future goals can boost motivation and the actual behavior to reach them, regardless 
of whether these goals are described in mildly or highly pessimistic terms. In keeping with this interpretation, we suggest that the time underestimation pattern reported in AN might be interpreted as the sign of a temporal proximity bias, which might affect their decisions for perspective goals (i.e., the weight loss) or outcomes they are interested in. Importantly, the absence of any correlation with the examined clinical, cognitive, and demographic measures corroborates the suggestion that the AN timing alteration documented in our study might be a symptomatic trait of this psychiatric condition, rather than being the epiphenomenon of other related deficits. Moreover, the evidence of a temporal underestimation in AN suggests that the reduced temporal discounting reported in previous work [e.g., Ref. (8)] might not be a hallmark of this clinical population, but a secondary effect of such timing bias. This is in line with previous evidence in healthy humans [e.g., Ref. (20)] reporting lower choice percentage for the smaller-immediate reward in participants who tended to underestimate time.

There are limitations in this study that should be mentioned. No information about the sub-type of AN condition (restrictive or purging) was available from the database. This would have been relevant in terms of exploring the possible contribution of impulsivity, as this is known to differ between the two subtypes of AN (79) and can affect timing performance (80). The timing task adopted in our current study did not include AN disorderspecific stimuli (i.e., picture of food stimuli) and/or other rewardrelated conditions. This would be another interesting aspect to explore in future investigations, as it might clarify whether and how the exposure to a primary reward, which may be perceived as an aversive experience in $\mathrm{AN}(81,82)$, affects time processing. We anticipate that an overestimation response might be predicted in the timing performance of $\mathrm{AN}$ in the presence of food stimuli, in line with the evidence of temporal overestimation in response to negative, stressful/fearful outcomes and anxiety [e.g., Ref. $(24,83-85)]$. The absence of measures to establish the temporal discounting response and the interoceptive awareness in our AN participants do not allow us to verify whether the reported temporal underestimation pattern is directly related with these measures, as it can be inferred from previous works in this field [e.g., Ref. $(20,26)]$. Therefore, the role of temporal discounting and interoception need to be explicitly tested in future investigations.

Strengths of our study are represented by the potential theoretical and clinical implications of the current discovery for the interpretation of AN disorder; the rigid inclusion/exclusion criteria; and the examination of demographic, clinical, and cognitive measures for the interpretation of the reported timing pattern.

\section{CONCLUSION}

The results of our study add new insights for the clinical interpretation of $\mathrm{AN}$, suggesting that the temporal underestimation

\section{REFERENCES}

1. Kaye WH, Wierenga CE, Bailer UF, Simmons AN, Bischoff-Grethe A. Nothing tastes as good as skinny feels: the neurobiology of anorexia nervosa. Trends Neurosci (2013) 36:110-20. doi:10.1016/j.tins.2013. 01.003 might contribute to the restrictive dietary pattern of this psychiatric disorder. In particular, we suggest that, because of a temporal underestimation bias, AN participants might perceive their future goals (i.e., the loss of further weight) as more proximal. This might contribute to the origin of their extreme motivation in pursuing a restrictive diet for a long time, as increasing the subjective temporal proximity of remote future goals can boost motivation and the actual behavior to reach them (78).

The absence of relationships between cognitive (WM and attention) and timing performance also suggests that the temporal underestimation bias of AN might be a symptomatic trait of this clinical condition. Moreover, the absence of such relationships, together with the absence of significant WM and attention deficits in this AN group, provide insights with regard to the possible neural underpinning of the reported timing alteration. In particular, one might speculate a main role of the insula cortex, over other neural regions, which activity is known to be altered in AN [Ref. (35), for a review]. This suggestion is provided in line with a recent study (86) which has dissociated the role of the insula activity in time processing from the role of basal ganglia for WM and attention processing. The potentially central role of the insula in the timing alteration of AN might make sense also of the food restriction habit of these patients, given the role of this region in regulating hunger/satiety signaling $(87,88)$.

Future investigations are required to extend this research in adult AN participants. The inclusion of other timing paradigms such as temporal expectation tasks [e.g., see Ref. (89-91), for a review], might allow us to more directly test the hypothesis that $\mathrm{AN}$ is affected by a temporal proximity bias for forthcoming goals.

\section{ETHICS STATEMENT}

All participants gave written informed consent during their recruitment. The study was approved by the Western Sydney Area Health Services Human Research Ethics Committee and by the University of Tasmania, School of Psychology, Research Ethics Committee.

\section{AUTHOR CONTRIBUTIONS}

Study concept and design, statistical analysis and interpretation of data, and critical revision of the manuscript: $\mathrm{CV}$ and KF. Acquisition of data and drafting of the manuscript: CV. All authors read and approved the version to be published.

\section{FUNDING}

This work has been supported by the University of Tasmania Fellowship and by the Alexander von Humboldt Research Fellowship for Experienced Researchers.

2. American Psychiatric Association. Diagnostic and Statistical Manual of Mental Disorders: Text Revision (DSM-IV-TR). 4th ed. Washington, DC: American Psychiatric Association (2000).

3. Hoek HW. Incidence, prevalence and mortality of anorexia nervosa and other eating disorders. Curr Opin Psychiatry (2006) 19:389-94. doi:10.1097/01. yco.0000228759.95237.78 
4. Arcelus J, Mitchell AJ, Wales J, Nielsen S. Mortality rates in patients with anorexianervosa and othereating disorders. Ameta-analysis of 36 studies. Arch Gen Psychiatry (2011) 68:724-31. doi:10.1001/archgenpsychiatry.2011.74

5. Birgegård A, Björck C, Norring C, Sohlberg S, Clinton D. Anorexic self-control and bulimic self-hate: differential outcome prediction from initial self-image. Int J Eat Disord (2009) 42:522-30. doi:10.1002/eat.20642

6. Bardone-Cone AM, WonderlichSA, Frost RO, BulikCM, MitchellJE, UppalaS, et al. Perfectionism and eating disorders: current status and future directions. Clin Psychol Rev (2007) 27:384-405. doi:10.1016/j.cpr.2006.12.005

7. Wade TD, Bulik CM, Prescott CA, Kendler KS. Sex influences on shared risk factors for bulimia nervosa and other psychiatric disorders. Arch Gen Psychiatry (2004) 61:251-6. doi:10.1001/archpsyc.61.3.251

8. Steinglass JE, Figner B, Berkowitz S, Simpson HB, Weber EU, Walsh BT. Increased capacity to delay reward in anorexia nervosa. J Int Neuropsychol Soc (2012) 18:773-80. doi:10.1017/S1355617712000446

9. Decker JH, Figner B, Steinglass JE. On weight and waiting: delay discounting in anorexia nervosa pretreatment and posttreatment. Biol Psychiatry (2015) 78:606-14. doi:10.1016/j.biopsych.2014.12.016

10. Steinglass JE, Lempert KM, Choo TH, Kimeldorf MB, Wall M, Walsh BT, et al. Temporal discounting across three psychiatric disorders: anorexia nervosa, obsessive compulsive disorder, and social anxiety disorder. Depress Anxiety (2017) 4:463-70. doi:10.1002/da.22586

11. Bartholdy S, Rennalls S, Danby H, Jacques C, Campbell IC, Schmidt U, et al. Temporal discounting and the tendency to delay gratification across the eating disorder spectrum. Eur Eat Disord Rev (2017) 25:344-50. doi:10.1002/ erv. 2513

12. King JA, Geisler D, Bernardoni F, Ritschel F, Böhm I, Seidel M, et al. Altered neural efficiency of decision making during temporal reward discounting in anorexia nervosa. J Am Acad Child Adolesc Psychiatry (2016) 55:972-9. doi:10.1016/j.jaac.2016.08.005

13. Ritschel F, King JA, Geisler D, Flohr L, Neidel F, Boehm I, et al. Temporal delay discounting in acutely ill and weight-recovered patients with anorexia nervosa. Psychol Med (2015) 45:1229-39. doi:10.1017/S0033291714002311

14. Ebert J, Prelec D. The fragility of time: time-insensitivity and valuation of the near and far future. Manage Sci (2007) 53:1423-38. doi:10.1287/ mnsc. 1060.0671

15. Killeen PR. An additive-utility model of delay discounting. Psychol Rev (2009) 16:602-19. doi:10.1037/a0016414

16. Takahashi $\mathrm{T}$. Loss of self-control in intertemporal choice may be attributable to logarithmic time-perception. Med Hypotheses (2005) 65:691-3. doi:10.1016/j.mehy.2005.04.040

17. Zauberman G, Kim BK, Malkoc SA, Bettman JR. Discounting time and time discounting: subjective time perception and intertemporal preferences. J Mark Res (2009) 46:543-56. doi:10.1509/jmkr.46.4.543

18. Kim BK, Zauberman G. Perception of anticipatory time in temporal discounting. J Neurosci Psychol Econ (2009) 2:91. doi:10.1037/a0017686

19. Baumann AA, Odum AL. Impulsivity, risk taking, and timing. Behav Process (2012) 90:408-14. doi:10.1016/j.beproc.2012.04.005

20. Suo T, Zhang F, Zhao G, Li H. The influence of time perception difference on intertemporal choice. Acta Psychol Sin (2014) 46:165-73. doi:10.3724/ SP.J.1041.2014.00165

21. Steward T, Mestre-Bach G, Vintró-Alcaraz C, Agüera Z, Jiménez-Murcia S, Granero R, et al. Delay discounting of reward and impulsivity in eating disorders: from anorexia nervosa to binge eating disorder. Eur Eat Disord $\operatorname{Rev}(2017)$ 25:601-6. doi:10.1002/erv.2543

22. Khalsa SS, Craske MG, Li W, Vangala S, Strober M, Feusner JD. Altered interoceptive awareness in anorexia nervosa: effects of meal anticipation, consumption and bodily arousal. Int JEat Disord (2015) 48:889-97. doi:10.1002/eat.22387

23. Wittmann M. Modulations of the experience of self and time. Conscious Cogn (2015) 38:172-81. doi:10.1016/j.concog.2015.06.008

24. Pollatos O, Laubrock J, Wittmann M. Interoceptive focus shapes the experience of time. PLoS One (2014) 9:e86934. doi:10.1371/journal.pone.0086934

25. Vicario CM, Kuran KA, Urgesi C. Does hunger sharpen senses? A psychophysics investigation on the effects of appetite in the timing of reinforcement-oriented actions. Psychol Res (2017). doi:10.1007/s00426-017-0934-y

26. Meissner K, Wittmann M. Body signals, cardiac awareness, and the perception of time. Biol Psychol (2011) 86(3):289-97. doi:10.1016/j. biopsycho.2011.01.001
27. Di Lernia D, Serino S, Pezzulo G, Pedroli E, Cipresso P, Riva G. Feel the time. Time perception as a function of interoceptive processing. Front Hum Neurosci (2018) 12:74. doi:10.3389/fnhum.2018.00074

28. Cazzato V, Mian E, Mele S, Tognana G, Todisco P, Urgesi C. The effects of body exposure on self-body image and esthetic appreciation in anorexia nervosa. Exp Brain Res (2016) 234:695-709. doi:10.1007/s00221-015-4498-z

29. Buhusi CV, Meck WH. Effect of clozapine on interval timing and working memory for time in the peak-interval procedure with gaps. Behav Processes (2007) 74:159-67. doi:10.1016/j.beproc.2006.10.004

30. Lewis PA, Miall RC. Remembering the time: a continuous clock. Trends Cogn Sci (2006) 10:401-6. doi:10.1016/j.tics.2006.07.006

31. Vicario CM, Martino D, Spata F, Defazio G, Giacchè R, Martino V, et al. Time processing in children with Tourette's syndrome. Brain Cogn (2010) 73:28-34. doi:10.1016/j.bandc.2010.01.008

32. Vicario CM. Cognitively controlled timing and executive functions develop in parallel? A glimpse on childhood research. Front Behav Neurosci (2013) 7:146. doi:10.3389/fnbeh.2013.00146

33. Vicario CM. Altered insula response to sweet taste processing in recovered anorexia and bulimia nervosa: a matter of disgust sensitivity? Am J Psychiatry (2013) 170:1497. doi:10.1176/appi.ajp.2013.13060748

34. Titova OE, Hjorth OC, Schiöth HB, Brooks SJ. Anorexia nervosa is linked to reduced brain structure in reward and somatosensory regions: a meta-analysis of VBM studies. BMC Psychiatry (2013) 13:110 doi:10.1186/1471-244X-13-110

35. Kaye WH, Fudge JL, Paulus M. New insights into symptoms and neurocircuit function of anorexia nervosa. Nat Rev Neurosci (2009) 10:573-84. doi: $10.1038 / \mathrm{nrn} 2682$

36. Shott ME, Pryor TL, Yang TT, Frank GK. Greater insula white matter fiber connectivity in women recovered from anorexia nervosa. Neuropsychopharmacology (2016) 41:498-507. doi:10.1038/npp.2015.172

37. Gaudio S, Wiemerslage L, Brooks SJ, Schiöth HB. A systematic review of resting-state functional-MRI studies in anorexia nervosa: evidence for functional connectivity impairment in cognitive control and visuospatial and body-signal integration. Neurosci Biobehav Rev (2016) 71:578-89. doi:10.1016/j.neubiorev.2016.09.032

38. Vicario CM, Rafal RD, Martino D, Avenanti A. Core, social and moral disgust are bounded: a review on behavioral and neural bases of repugnance in clinical disorders. Neurosci Biobehav Rev (2017) 80:185-200. doi:10.1016/j. neubiorev.2017.05.008

39. Ferrandez AM, Hugueville L, Lehéricy S, Poline JB, Marsault C, Pouthas V. Basal ganglia and supplementary motor area subtend duration perception: an fMRI study. Neuroimage (2003) 19:1532-44. doi:10.1016/ S1053-8119(03)00159-9

40. Maquet P, Lejeune H, Pouthas V, Bonnet M, Casini L, Macar F, et al. Brain activation induced by estimation of duration: a PET study. Neuroimage (1996) 3:119-26. doi:10.1006/nimg.1996.0014

41. Tregellas JR, Davalos DB, Rojas DC. Effect of task difficulty on the functional anatomy of temporal processing. Neuroimage (2006) 32:307-15. doi:10.1016/j.neuroimage.2006.02.036

42. Wiener M,Turkeltaub P, Coslett HB. Theimage of time: a voxel-wise meta-analysis. Neuroimage (2010) 49:1728-40. doi:10.1016/j.neuroimage.2009.09.064

43. Meck WH. Neuropharmacology of timing and time perception. Brain Res Cogn Brain Res (1998) 6:233. doi:10.1016/S0926-6410(97)00031-1

44. Meck WH. Frontal cortex lesions eliminate the clock speed effect of dopaminergic drugs on interval timing. Brain Res (2006) 1108:157-67. doi:10.1016/j. brainres.2006.06.046

45. Koch G, Costa A, Brusa L, Peppe A, Gatto I, Torriero S, et al. Impaired reproduction of second but not millisecond time intervals in Parkinson's disease. Neuropsychologia (2008) 46:1305-13. doi:10.1016/j. neuropsychologia.2007.12.005

46. Vicario CM, Gulisano M, Martino D, Rizzo R. Timing recalibration in childhood Tourette syndrome associated with persistent pimozide treatment. J Neuropsychol (2016) 10:211-22. doi:10.1111/jnp.12064

47. Frank GK, Bailer UF, Henry SE, Drevets W, Meltzer CC, Price JC, et al. Increased dopamine D2/D3 receptor binding after recovery from anorexia nervosa measured by positron emission tomography and [11c] raclopride Biol Psychiatry (2005) 58:908-12. doi:10.1016/j.biopsych.2005.05.003

48. Montague PR, Hyman SE, Cohen JD. Computational roles for dopamine in behavioural control. Nature (2004) 431:760-7. doi:10.1038/nature03015 
49. Wittmann M, Leland DS, Churan J, Paulus MP. Impaired time perception and motor timing in stimulant-dependent subjects. Drug Alcohol Depend (2007) 90:183-92. doi:10.1016/j.drugalcdep.2007.03.005

50. Hinton SC, Meck WH. Frontal-striatal circuitry activated by human peak-interval timing in the supra-seconds range. Brain Res Cogn Brain Res (2004) 21:171-82. doi:10.1016/j.cogbrainres.2004.08.005

51. Frank GK, Reynolds JR, Shott ME, Jappe L, Yang TT, Tregellas JR, et al. Anorexia nervosa and obesity are associated with opposite brain reward response. Neuropsychopharmacology (2012) 37:2031-46. doi:10.1038/npp.2012.51

52. Faulkner KK, Duecker SJ. Stress, time distortion, and failure to recover among obese individuals: implications for weight-gain and dieting. Int J Eat Disord (1989) 8(2):247-50. doi:10.1002/1098-108X(198903)8:2<247::AIDEAT2260080217>3.0.CO;2-V

53. Merson F, Perriot J. Social deprivation and time perception, the impact on smoking cessation. Sante Publique (2011) 23:359-70. doi:10.3917/ spub.115.0359

54. Sayette MA, Loewenstein G, Kirchner TR, Travis T. Effects of smoking urge on temporal cognition. Psychol Addict Behav (2005) 19:88-93. doi:10.1037/0893-164X.19.1.88

55. Turel O, Brevers D, Bechara A. Time distortion when users at-risk for social media addiction engage in non-social media tasks. J Psychiatr Res (2018) 97:84-8. doi:10.1016/j.jpsychires.2017.11.014

56. Casini L, Ivry RB. Effects of divided attention on temporal processing in patients with lesions of the cerebellum or frontal lobe. Neuropsychology (1999) 13:10-21. doi:10.1037/0894-4105.13.1.10

57. Enns JT, Brehaut JC, Shore DI. The duration of a brief event in the mind's eye. J Gen Psychol (1999) 126:355-72. doi:10.1080/00221309909595371

58. Block RA, Zakay D. Prospective and retrospective duration judgments: a meta-analytic review. Psychon Bull Rev (1997) 4:184-97. doi:10.3758/ BF03209393

59. Tse PU, Intriligator J, Rivest J, Cavanagh P. Attention and the subjective expansion of time. Percept Psychophys (2004) 6:1171-89. doi:10.3758/ BF03196844

60. Vicario CM, Rappo G, Pepi AM, Oliveri M. Timing flickers across sensory modalities. Perception (2009) 38:1144-51. doi:10.1068/p6362

61. Vicario CM, Martino D, Pavone EF, Fuggetta G. Lateral head turning affects temporal memory. Percept Mot Skills (2011) 113:3-10. doi:10.2466/04.22. PMS.113.4.3-10

62. Vicario CM, Bonní S, Koch G. Left hand dominance affects supra-second time processing. Front Integr Neurosci (2011) 5:65. doi:10.3389/fnint.2011.00065

63. Mioni G, Stablum F, Prunetti E, Grondin S. Time perception in anxious and depressed patients: a comparison between time reproduction and time production tasks. JAffect Disord (2016) 196:154-63. doi:10.1016/j. jad.2016.02.047

64. Williams LM, Simms E, Clark CR, Paul RH, Rowe D, Gordon E. The test-retest reliability of a standardized neurocognitive and neurophysiological test battery: "neuromarker". Int J Neurosci (2005) 115:1605-30. doi:10.1080/00207450590958475

65. Clark CR, Paul RH, Williams LM, Arns M, Fallahpour K, Handmer C, et al. Standardized assessment of cognitive functioning during development and aging using an automated touchscreen battery. Arch Clin Neuropsychol (2006) 21:449-67. doi:10.1016/j.acn.2006.06.005

66. Paul RH, Gunstad J, Cooper N, Williams LM, Clark CR, Cohen RA, et al. Cross-cultural assessment of neuropsychological performance and electrical brain function measures: additional validation of an international brain database. Int J Neurosci (2007) 117:549-68. doi:10.1080/00207450600773665

67. Hebebrand J, Wehmeier PM, Remschmidt H. Weight criteria for diagnosis of anorexia nervosa. Am J Psychiatry (2000) 157:1024. doi:10.1176/appi. ajp.157.6.1024

68. Garner DM. EDI-3 Eating Disorder Inventory-3: Professional Manual. Odessa, FL: Psychological Assessment Resources Inc. (2004).

69. Baddeley A. The episodic buffer: a new component of working memory? Trends Cogn Sci (2000) 4:417-23. doi:10.1016/S1364-6613(00)01538-2

70. Monsell S. Task switching. Trends Cogn Sci (2003) 7:134-40. doi:10.1016/ S1364-6613(03)00028-7

71. Lovibond PF, Lovibond SH. The structure of negative emotional states: comparison of the depression anxiety stress scales (DASS) with the beck depression and anxiety inventories. Behav Res Ther (1995) 33:335-42. doi:10.1016/0005-7967(94)00075-U
72. Block RA, Zakay D, Hancock PA. Developmental changes in human duration judgments: a meta-analytic review. $\operatorname{Dev} \operatorname{Rev}(1999)$ 19:183-211. doi:10.1006/ drev.1998.0475

73. Gunstad J, Cohen RA, Paul RH, Luyster FS, Gordon E. Age effects in time estimation: relationship to frontal brain morphometry. JIntegr Neurosci (2006) 5:75-87. doi:10.1142/S0219635206001045

74. Schneider BA, Avivi-Reich M, Mozuraitis M. A cautionary note on the use of the Analysis of Covariance (ANCOVA) in classification designs with and without within-subject factors. Front Psychol (2015) 6:474. doi:10.3389/ fpsyg.2015.00474

75. Simes RJ. An improved Bonferroni procedure for multiple tests of significance. Biometrika (1986) 73:751-4. doi:10.1093/biomet/73.3.751

76. Pollatos O, Herbert BM, Schandry R, Gramann K. Impaired central processing of emotional faces in anorexia nervosa. Psychosom Med (2008) 70:701-8. doi:10.1097/PSY.0b013e31817e41e6

77. Pollatos O, Kurz AL, Albrecht J, Schreder T, Kleemann AM, Schöpf V, et al. Reduced perception of bodily signals in anorexia nervosa. Eat Behav (2008) 9:381-8. doi:10.1016/j.eatbeh.2008.02.001

78. Bashir NY, Wilson AE, Lockwood P, Chasteen AL, Alisat S. The time for action is now: subjective temporal proximity enhances pursuit of remote-future goals. Soc Cogn (2014) 32:83-93. doi:10.1521/soco.2014.32.1.83

79. Lavender JM, Mitchell JE. Eating disorders and their relationship to impulsivity. Curr Treat Options Psychiatry (2015) 2:394-401. doi:10.1007/ s40501-015-0061-6

80. Wittmann M, Paulus MP. Decision making, impulsivity and time perception. Trends Cogn Sci (2008) 12:7-12. doi:10.1016/j.tics.2007.10.004

81. Cowdrey FA, Park RJ, Harmer CJ, McCabe C. Increased neural processing of rewarding and aversive food stimuli in recovered anorexia nervosa. Biol Psychiatry (2011) 70:736-43. doi:10.1016/j.biopsych.2011.05.028

82. Vicario CM, Crescentini C. Punishing food: what brain activity can tell us about the representation of food in recovered anorexia nervosa. Biol Psychiatry (2012) 71:e31-2. doi:10.1016/j.biopsych.2011.10.036

83. Gil S, Niedenthal PM, Droit-Volet S. Anger and time perception in children. Emotion (2007) 7:219-25. doi:10.1037/1528-3542.7.1.219

84. Bar-Haim Y, Kerem A, Lamy D, Zakay D. When time slows down: the influence of threat on time perception in anxiety. Cogn Emot (2010) 24:255-63. doi:10.1080/02699930903387603

85. Vicario CM, Felmingham K. Slower time estimation in post-traumatic stress disorder. Sci Rep (2018) 8:392. doi:10.1038/s41598-017-18907-5

86. Üstün S, Kale EH, Çiçek M. Neural networks for time perception and working memory. Front Hum Neurosci (2017) 11:83. doi:10.3389/fnhum.2017.00083

87. Tataranni PA, Gautier JF, Chen K, Uecker A, Bandy D, Salbe AD, et al. Neuroanatomical correlates of hunger and satiation in humans using positron emission tomography. Proc Natl Acad Sci U S A (1999) 96:4569-74. doi:10.1073/pnas.96.8.4569

88. Del Parigi A, Chen K, Gautier JF, Salbe AD, Pratley RE, Ravussin E, et al. Sex differences in the human brain's response to hunger and satiation. Am J Clin Nutr (2002) 75(6):1017-22. Erratum in: Am J Clin Nutr (2002) 76(2):492. doi:10.1093/ajcn/75.6.1017

89. Avanzino L, Martino D, Martino I, Pelosin E, Vicario CM, Bove M, et al. Temporal expectation in focal hand dystonia. Brain (2013) 136:444-54. doi:10.1093/brain/aws328

90. Martino D, Lagravinese G, Pelosin E, Chaudhuri RK, Vicario CM, Abbruzzese G, et al. Temporal processing of perceived body movement in cervical dystonia. Mov Disord (2015) 30:1005-7. doi:10.1002/mds.26225

91. Avanzino L, Pelosin E, Vicario CM, Lagravinese G, Abbruzzese G, Martino D. Time processing and motor control in movement disorders. Front Hum Neurosci (2016) 10:631. doi:10.3389/fnhum.2016.00631

Conflict of Interest Statement: The authors declare that the research was conducted in the absence of any commercial or financial relationships that could be construed as a potential conflict of interest.

Copyright $(2018$ Vicario and Felmingham. This is an open-access article distributed under the terms of the Creative Commons Attribution License (CC BY). The use, distribution or reproduction in other forums is permitted, provided the original author(s) and the copyright owner are credited and that the original publication in this journal is cited, in accordance with accepted academic practice. No use, distribution or reproduction is permitted which does not comply with these terms. 